PROCEEDINGS OF THE

AMERICAN MATHEMATICAL SOCIETY

Volume 137, Number 9, September 2009, Pages 2857-2864

S 0002-9939(09)09853-0

Article electronically published on March 25, 2009

\title{
A NOTE ON THE STRONG DIRECT SUMMAND CONJECTURE
}

\author{
JASON MCCULLOUGH
}

(Communicated by Bernd Ulrich)

\begin{abstract}
We prove special cases of the strong direct summand conjecture and the related strong monomial conjecture.
\end{abstract}

\section{INTRODUCTION}

In HH95, Hochster and Huneke formulated the following conjecture.

Conjecture 1.1 (Vanishing Maps of Tor Conjecture (VMTC)). Let $R \rightarrow A \rightarrow S$ be homomorphisms of Noetherian rings such that $R$ and $S$ are regular and such that $A$ is module-finite over $R$. Let $M$ be any $R$-module. Then the maps

$$
\operatorname{Tor}_{i}^{R}(M, A) \rightarrow \operatorname{Tor}_{i}^{R}(M, S)
$$

are zero for all $i \geq 1$.

Hochster and Huneke show that the VMTC is a consequence of the existence of weakly functorial big Cohen-Macaulay algebras and, therefore, a theorem in the equicharacteristic case. The VMTC also holds in dimension at most 3 by Hochster's extension [Hoc02 of Heitmann's theorem in Hei02. Hochster and Huneke proved similar results previously in [HH90 and [HH93 using tight closure theory. Ranganathan proved some special cases in [Ran00]. The study of this conjecture is motivated by the fact that the VMTC implies that direct summands of regular rings are Cohen-Macaulay and that the VMTC implies Hochster's direct summand conjecture (see [HH90, HH93, [HH95]).

Conjecture 1.2 (Direct Summand Conjecture (DSC)). Let $R$ be a regular ring and let $A$ be a module-finite extension of $R$. Then $R$ is a direct summand of $A$ as an $R$-module.

The DSC first appeared in Hoc73, where Hochster proved the equicharacteristic case. The DSC and its equivalent forms (including the monomial conjecture (MC), the canonical element conjecture and the improved new intersection conjecture) have been studied by many people. We refer the reader to [Hoc83, [Koh86], Got83, [EG81, Dut01], and Rob76]. Like the other homological conjectures, the VMTC is still an open question in the general mixed characteristic case.

In [Ran00, Ranganathan proved that the VMTC is actually equivalent to the following statement.

Received by the editors February 25, 2008, and, in revised form, November 27, 2008.

2000 Mathematics Subject Classification. Primary 13D22; Secondary 13D45, 13D07, 13H05.

Key words and phrases. Strong direct summand conjecture, strong monomial conjecture, vanishing maps of tor, homological conjectures.

(C)2009 American Mathematical Society Reverts to public domain 28 years from publication 2857 
Conjecture 1.3 (Strong Direct Summand Conjecture (SDSC)). Let $(R, \mathfrak{m})$ be a regular local ring and let $A$ be a module-finite extension. Let $Q$ be a height one prime ideal of $A$ containing $x R$, where $x$ is a minimal generator of $\mathfrak{m}$. Then $x R$ is a direct summand of $Q$.

By the equivalence, it follows that the SDSC is a theorem in the equicharacteristic case and in dimension at most three. The equivalence is proved through a number of reductions and equivalences. See Hoc07 for a nice summary. As the name suggests, the SDSC implies the direct summand conjecture. Both conjectures reduce easily to the case where $R$ and $A$ are complete local domains. So it is natural to formulate a version of the monomial conjecture that implies the SDSC. Ranganathan formulated the following strong monomial conjecture in Ran00:

Conjecture 1.4 (Strong Monomial Conjecture (SMC)). Let $A$ be a local domain with system of parameters $x_{1}, \ldots, x_{d}$. Let $Q$ be a height one prime of $A$ containing $x_{1}$. Then

$$
x_{1}\left(x_{1} \cdots x_{d}\right)^{t} \notin\left(x_{1}^{t+1}, \ldots, x_{d}^{t+1}\right) Q \text { for all } t>0 .
$$

The SMC is one of the main focuses of this paper. Moreover, we extend the notion of the SMC to the following setting. Let $A$ be a local domain with system of parameters $\mathbf{x}=x_{1}, \ldots, x_{d}$. Let $Q$ be a height one prime of $A$ and let $z \in Q-\mathbf{x} Q$. Then we say the SMC holds for $A, Q, \mathbf{x}$ and $z$ if

$$
z\left(x_{1} \cdots x_{d}\right)^{t} \notin\left(x_{1}^{t+1}, \ldots, x_{d}^{t+1}\right) Q \text { for all } t>0 .
$$

Hence the usual SMC is the case where $z=x_{1}$. The generality introduced is necessary later in the statement of Theorem 2.5.

We extend results of Dutta Dut98] and Strooker and Stückrad SS93] to prove the following:

Proposition 2.1. Let $A$ be a complete local domain and let $\mathbf{x}=x_{1}, \ldots, x_{d}$ be a system of parameters for $A$. Let $Q$ be a height one prime of $A$. Let $z \in Q-\mathbf{x} Q$. Let $(S, \mathfrak{n}, k)$ be a local complete intersection ring with $A=S / \mathfrak{p}, \operatorname{dim}(S)=\operatorname{dim}(A)$ and $\mathfrak{p} S_{\mathfrak{p}}=0$. Then

$$
z\left(x_{1} \cdots x_{d}\right)^{t} \notin\left(x_{1}^{t+1}, \ldots, x_{d}^{t+1}\right) Q \text { for all } t>0
$$

if and only if $\operatorname{Im}\left(\operatorname{Hom}_{S}(Q, S) \stackrel{z}{\longrightarrow} \operatorname{Hom}_{S}(S, S)=S\right)$ is not contained in $\mathbf{x} S$. In particular, the strong monomial conjecture holds if and only if

$$
\operatorname{Im}\left(\operatorname{Hom}_{S}(Q, S) \stackrel{x_{1}}{\longrightarrow} \operatorname{Hom}_{S}(S, S)\right) \not \subset \mathbf{x} S .
$$

Here $\operatorname{Hom}_{S}(Q, S) \stackrel{z}{\longrightarrow} \operatorname{Hom}_{S}(S, S)$ is the map induced by applying $\operatorname{Hom}_{S}(-, S)$ to the map $S \rightarrow Q$ sending $1 \mapsto z$. Using the previous result, we obtain the following corollary.

Corollary 2.2. The Strong Monomial Conjecture holds for all local rings if and only if it holds for all local almost complete intersection rings.

Combining our results with one from [Dut98, we also prove the following characterization of the SMC in terms of the length of certain Koszul homology modules. Here $S$ denotes a complete intersection ring mapping onto $A$ as in Proposition 2.1.

Proposition 2.3. Set $J=\operatorname{Im}\left(\operatorname{Hom}_{S}(Q, S) \stackrel{z}{\longrightarrow} \operatorname{Hom}_{S}(S, S)\right) \subset S$. Then the $S M C$ holds for $Q, z$ and $\mathbf{x}$ if and only if $\ell(J / \mathbf{x} J)>\ell\left(H_{1}(\mathbf{x} ; S / J)\right)$. 
We show that under the mild hypothesis that $A / \mathfrak{n}$ is infinite where $\mathfrak{n}$ is the maximal ideal of $A$, we can make use of Samuel's theory of superficial elements and the previous result to show the following:

Theorem 2.5. Suppose the residue field of $A$ is infinite. Let $x_{1}, \ldots, x_{d}$ be a system of parameters. Let $Q$ be a height one prime in $A$. Let $z \in Q$. Then there exist $y_{1}, \ldots, y_{d-1} \in A$ such that $\left(x_{1}, \ldots, x_{d}\right)=\left(y_{1}, \ldots, y_{d-1}, x_{d}\right)$ and such that the SMC holds for $A, Q, y_{1}, \ldots, y_{d-1}, x_{d}^{t}$ and $x_{1}$ for $t \gg 0$.

Note that $x_{1}, \ldots, x_{d}$ satisfy the SMC if and only if $y_{1}, \ldots, y_{d-1}, x_{d}$ as above do. The above results are extensions of results of Dutta on the monomial conjecture in Dut98 and use similar methods.

Finally, we show that the SDSC is true if one assumes the direct summand conjecture in lower dimension and that $H_{\mathfrak{m}}^{d-1}(A)=0$, where $d=\operatorname{dim}(A)$. We note that this case includes rings $A$ that are Cohen-Macaulay, local almost complete intersection domains, and complete local normal domains with canonical module $S_{3}$. (See Dut97.)

\section{The Strong Monomial CONJECTURE}

In [SS93, Strooker and Stückrad characterize the monomial conjecture in the following way. They show that the monomial conjecture holds for all rings if, for all complete intersection rings $S$ and all ideals of zerodivisors $\mathfrak{a}, \operatorname{Ann}_{S}(\mathfrak{a})$ is not contained in any parameter ideal of $S$. Dutta Dut98 proved a similar result along with further statements regarding the MC. We extend these results to the strong monomial conjecture below.

For this section, let us fix some notation. Let $(R, \mathfrak{m})$ denote a regular local ring with $\mathfrak{m}$ its unique maximal ideal. Let $A$ denote a module-finite extension of $R$. Let $d=\operatorname{dim}(A)=\operatorname{dim}(R)$. For the purposes of the SDSC and SMC, we may assume that $R$ and $A$ are complete local domains. We focus on the SMC for most of this section.

We also make use of the following setup. Let $R$ and $A$ be as above. By mapping a Cohen ring onto $A$ and modding out by an appropriate regular sequence, one can construct a complete intersection $S$ surjecting onto $A$ such that $A=S / \mathfrak{p}$ for a prime $\mathfrak{p} \subset S$ with $\mathfrak{p} S_{\mathfrak{p}}=(0)$ and $\mathfrak{p} \in \operatorname{Ass}_{S}(S)$. See Proposition 1 in SS93. For $Q$ a height one ideal in $A$ and $z \in Q$, we denote by $\operatorname{Hom}_{S}(Q, S) \stackrel{z}{\longrightarrow} \operatorname{Hom}_{S}(S, S)$ the map obtained by applying $\operatorname{Hom}_{S}(-, S)$ to the map $S \rightarrow Q$ sending $1 \mapsto z$. Finally, we abbreviate "system of parameters" by s.o.p.

Proposition 2.1. Let $A$ be a complete local domain and let $\mathbf{x}=x_{1}, \ldots, x_{d}$ be an s.o.p. for $A$. Let $Q$ be a height one prime of $A$. Let $z \in Q-\mathbf{x} Q$. Let $(S, \mathfrak{n})$ be a local complete intersection ring with $A=S / \mathfrak{p}$ and $\operatorname{dim}(S)=\operatorname{dim}(A)$. Then,

$$
z\left(x_{1} \cdots x_{d}\right)^{t} \notin\left(x_{1}^{t+1}, \ldots, x_{d}^{t+1}\right) Q \text { for all } t>0
$$

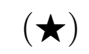

if and only if $\operatorname{Im}\left(\operatorname{Hom}_{S}(Q, S) \stackrel{z}{\longrightarrow} \operatorname{Hom}_{S}(S, S)=S\right)$ is not contained in $\mathbf{x} S$. In particular, the strong monomial conjecture holds if and only if

$$
\operatorname{Im}\left(\operatorname{Hom}_{S}(Q, S) \stackrel{x_{1}}{\longrightarrow} \operatorname{Hom}_{S}(S, S)\right) \not \subset \mathbf{x} S .
$$

Proof. We have the sequence of maps

$$
S \rightarrow A \stackrel{z}{\longrightarrow} Q .
$$


Set $E=E_{S}(k) \simeq H_{\mathfrak{n}}^{d}(S)$, where $E_{S}(k)$ is the injective envelope of the residue field of $S$. Applying $H_{\mathfrak{n}}^{d}(-)$ and $-\otimes_{S} S / \mathbf{x} S$ we get the following diagram:

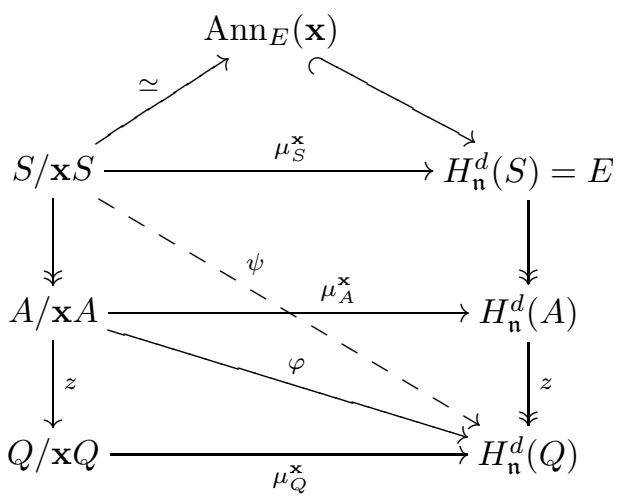

In this diagram, $\mu_{M}^{\mathbf{x}}: M \rightarrow H_{\mathfrak{n}}^{d}(M)$ denotes the direct limit map

$$
\mu_{M}^{\mathbf{x}}: M / \mathbf{x} M \rightarrow \underset{t}{\lim } M / \mathbf{x}^{t} M \simeq H_{\mathfrak{n}}^{d}(M) .
$$

Note that $\mu_{S}^{\mathrm{x}}$ is injective because $S$ is Cohen-Macaulay and that $\mu_{S}^{\mathrm{x}}$ factors through $\operatorname{Ann}_{E}(\mathbf{x})$. Also note that the statement $(\star)$ holds if and only if $\varphi \neq 0$. Further, since $S / \mathrm{x} S \rightarrow A / \mathrm{x} A$ is surjective, $\varphi \neq 0$ if and only if $\psi \neq 0$.

Let $(-)^{\vee}=\operatorname{Hom}_{S}(-, E)$ denote the Matlis dual. Applying $(-)^{\vee}$ to the above diagram yields the following commutative diagram:

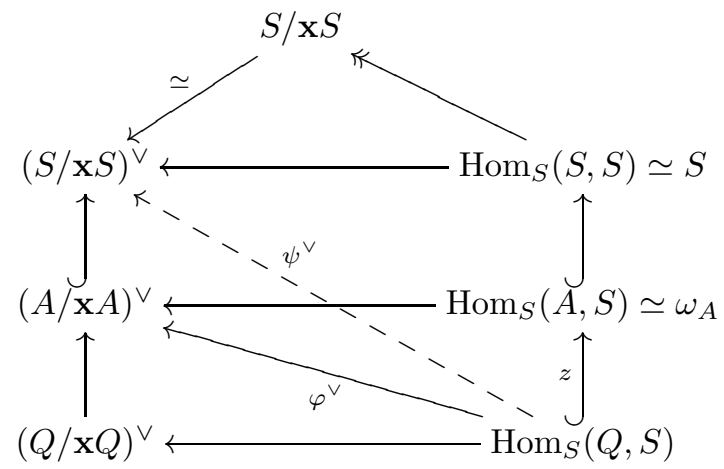

Since $(-)^{\vee}$ is a faithful, exact functor, we have that $\psi \neq 0$ if and only if $\psi^{\vee} \neq$ 0 . Since $\operatorname{Hom}_{S}(Q, S)$ injects into $S$, it can be seen that this is equivalent to $\operatorname{Im}\left(\operatorname{Hom}_{S}(Q, S)\right) \not \subset \mathbf{x} S$.

As noted in the introduction, the freedom in separating $z$ from $x_{1}$ in the previous proposition will be used to prove an analog of the SMC later in Theorem 2.5.

We follow the development by Dutta Dut98 for extending results about the monomial conjecture to the SMC. First, we obtain the following reduction of the SMC using the previous result.

Corollary 2.2. The Strong Monomial Conjecture holds for all local rings if and only if it holds for all local almost complete intersection rings.

Proof. Let $A$ be a local ring and let $S$ be a complete intersection ring of equal dimension with $A=S / \mathfrak{p}, \mathfrak{p} S_{\mathfrak{p}}=0$, and $\mathfrak{p} \in \operatorname{Ass}_{S}(S)$. Let $Q$ be a height one prime 
of $A$. Pick $\lambda \in \mathfrak{p}-\bigcup\left\{\mathfrak{q} \in \operatorname{Ass}_{S}(S), \mathfrak{q} \neq \mathfrak{p}\right\}$. Write $Q=\widetilde{Q} / \mathfrak{p}$ for a height one prime $\widetilde{Q}$ of $S$. Now take $\mathfrak{q} \in \operatorname{Ass}_{S}(S)$. If $\mathfrak{q}=\mathfrak{p}$, then since $\mathfrak{p} S_{\mathfrak{p}}=0$, we have $\mathfrak{q} \notin \operatorname{Supp}_{S}(\mathfrak{p} / \lambda S)$. If $\mathfrak{q} \neq \mathfrak{p}$, then $\mathfrak{q} \notin \operatorname{Supp}_{S}(\mathfrak{p} / \lambda S)$ by the choice of $\lambda$. Hence $\operatorname{Ass}_{S}\left(\operatorname{Hom}_{S}(\mathfrak{p} / \lambda S, S)\right)=\operatorname{Supp}_{S}(\mathfrak{p} / \lambda S) \cap \operatorname{Ass}_{S}(S)=\varnothing$ and so $\operatorname{Hom}_{S}(\mathfrak{p} / \lambda S, S)=0$. Therefore the injection $\varphi$ from the exact sequence

$$
0 \rightarrow \operatorname{Hom}_{S}(Q, S) \stackrel{\varphi}{\longrightarrow} \operatorname{Hom}_{S}(\widetilde{Q} / \lambda S, S) \rightarrow \operatorname{Hom}_{S}(\mathfrak{p} / \lambda S, S)
$$

is actually an isomorphism. Hence the image of $\operatorname{Hom}_{S}(Q, S)$ in $S$ is the same as the image of $\operatorname{Hom}_{S}(\widetilde{Q} / \lambda S, S)$. The claim follows from the previous proposition.

In the following result, $H_{i}(\mathbf{x} ; M)$ denotes the $i$ th Koszul homology of the $A$ module $M$ with respect to a list of elements $\mathbf{x}$.

Proposition 2.3. Let $A$ be a complete local domain and let $S$ be a complete intersection surjecting onto $A$ with $A=S / \mathfrak{p}$ as in Proposition 2.1. Let $Q$ be a height one prime of $A$. Set $J=\operatorname{Im}\left(\operatorname{Hom}_{S}(Q, S) \stackrel{z}{\longrightarrow} \operatorname{Hom}_{S}(S, S)\right) \subset S$. Then the Strong Monomial Conjecture holds for $Q, z$ and $\mathbf{x}$ if and only if $\ell(J / \mathbf{x} J)>\ell\left(H_{1}(\mathbf{x} ; S / J)\right)$.

Proof. Consider the short exact sequence

$$
0 \rightarrow J \rightarrow S \rightarrow S / J \rightarrow 0
$$

Applying $-\otimes_{S} S / \mathrm{x} S$, we get the exact sequence

$$
0 \rightarrow H_{1}(\mathbf{x} ; S / J) \rightarrow J / \mathbf{x} J \rightarrow S / \mathbf{x} S \rightarrow S /(J+x S) \rightarrow 0 .
$$

By Proposition 2.1. the SMC holds if and only if $J \not \subset \mathrm{x} S$, which happens if and only if $\ell(S / \mathbf{x} S)>\ell(S /(J+\mathbf{x} S))$. (Here $\ell(M)$ denotes the length of $M$.) Since length is additive, we have $\ell(J / \mathbf{x} J)>\ell\left(H_{1}(\mathbf{x} ; S / J)\right)$ and conversely.

We will make use of the following result of Dutta, which appears as Proposition 1.4 in Dut98. Here $e\left(x_{1}, \ldots, x_{n} ; A\right)$ denotes the Hilbert multiplicity of $A$ with respect to an s.o.p. $x_{1}, \ldots, x_{n}$.

Proposition 2.4 (Dutta). Let $x_{1}, \ldots, x_{n}$ be an s.o.p. of a local ring $A$ such that

$$
e\left(x_{1}, \ldots, x_{n} ; A\right)=e\left(x_{2}, \ldots, x_{n} ; A / x_{1} A\right)=\cdots=e\left(x_{n} ; A /\left(x_{1}, \ldots, x_{n-1}\right) A\right) .
$$

Then $H_{i}\left(x_{1}, \ldots, x_{n-1} ; A\right)$ is a module of finite length for every $i>0$.

By extending by a faithfully flat extension, we may assume that the residue field of $A$ is infinite. Recall that this does not change the issue of splitting. So we may apply Samuel's theory of superficial elements. We refer the reader to ZS93 for background material on superficial elements.

Theorem 2.5. Suppose the residue field of $A$ is infinite. Let $x_{1}, \ldots, x_{d}$ be an s.o.p. Let $Q$ be a height one prime in $A$. Then there exist $y_{1}, \ldots, y_{d-1} \in A$ such that $\left(x_{1}, \ldots, x_{d-1}\right)=\left(y_{1}, \ldots, y_{d-1}\right)$ and such that the SMC holds for $A, Q$, $y_{1}, \ldots, y_{d-1}, x_{d}^{t}$ and $x_{1}$ for $t \gg 0$.

Proof. Let $S$ be as in Proposition 2.3, Lift the system of parameters $x_{1}, \ldots, x_{d}$ to a system of parameters in $S$. (By an abuse of notation, we write them the same.) Thus by the remark to Theorem VIII-22 of [ZS93, we may choose $y_{1}, \ldots, y_{d-1}$ in such a way that each $y_{i}$ is of the form

$$
y_{i}=a_{1 j} x_{1}+\cdots+\lambda_{i} x_{i}+\cdots a_{d j} x_{d}
$$


with $\lambda_{i}$ a unit and $a_{i j} \in A$ for all $i$ and $j$. (Note that $\left(x_{1}, \ldots, x_{d}\right)=\left(y_{1}, \ldots, y_{d-1}, x_{d}\right)$ in $S$ and hence also in $A$.) Further, we may choose the $y_{i}$ to be superficial elements with respect to $\mathbf{x}$. The same is true of their images in $S / J$, where $J=\operatorname{Im}\left(\operatorname{Hom}_{S}(Q, S) \stackrel{x_{1}}{\longrightarrow} \operatorname{Hom}_{S}(S, S)\right)$ as above. We have by Proposition 2.4 that $\ell\left(H_{1}\left(y_{1}, \ldots, y_{d-1} ; S / J\right)\right)<\infty$.

Set $\mathbf{y}=y_{1}, \ldots, y_{d-1}$. Now consider the exact sequence

$$
0 \rightarrow \frac{H_{1}(\mathbf{y} ; S / J)}{x_{d}^{t} H_{1}(\mathbf{y} ; S / J)} \rightarrow H_{1}\left(y_{1}, \ldots, y_{d-1}, x_{d}^{t} ; S / J\right) \rightarrow\left(0: x_{d}^{t}\right) A / \mathbf{y} \rightarrow 0 .
$$

Since $\ell\left(H_{1}(\mathbf{y} ; S / J)\right)<\infty$, we have $x_{n}^{t} H_{1}(\mathbf{y} ; A)=0$ for $t \gg 0$. Also $\ell\left(\left(0: x_{n}^{t}\right) A / \mathbf{y}\right)$ is constant for $t \gg 0$. Therefore $l\left(H_{1}\left(y_{1}, \ldots, y_{d-1}, x_{d}^{t} ; S / J\right)\right)$ is constant for $t \gg 0$.

Finally, observe that $\operatorname{since} \operatorname{depth}(S)=d$, we have $\operatorname{dim}(J)=d$. Thus

$$
\lim _{t \rightarrow \infty} J /\left(y_{1}, \ldots, y_{d-1}, x_{d}^{t}\right) J=\infty .
$$

It then follows that for $t$ sufficiently large,

$$
\ell\left(J /\left(y_{1}, \ldots, y_{d-1}, x_{d}^{t}\right) J\right)>\ell\left(H_{1}\left(y_{1}, \ldots, y_{d-1}, x_{d}^{t} ; S / J\right)\right) .
$$

By the previous proposition, the SMC holds for $x_{1}, Q$ and the system of parameters $y_{1}, \ldots, y_{d-1}, x_{d}^{t}$.

Now we return to the Strong Direct Summand Conjecture. In Theorem 2.1 of Dut01, Dutta proved that the Direct Summand Conjecture holds when $H_{\mathfrak{m}}^{d-1}(A)=$ 0 . (To be precise, it is shown that the stronger canonical element conjecture holds for A.) We use this result to show that the SDSC also holds in this case if one assumes the general Direct Summand Conjecture in lower dimension.

Theorem 2.6. Let $R$ and $A$ be complete local rings such that $R$ is regular and such that $A$ is module-finite over $R$. Suppose $H_{\mathfrak{m}}^{d-1}(A)=0$ and that the Direct Summand Conjecture holds in dimension $d-1$. Then the Strong Direct Summand Conjecture holds for A.

Proof. Let $Q$ be a height one prime of $A$ lying over $x R$ where $x$ is a regular parameter of $R$. Note that by [Dut97], since $H_{\mathfrak{m}}^{d-1}(A)=0$, the Direct Summand Conjecture holds for $A$. This is equivalent to saying that the map $\operatorname{Hom}_{R}(A, R) \rightarrow \operatorname{Hom}_{R}(R, R)$ is onto. By local duality, this is equivalent to the map $H_{\mathfrak{m}}^{d}(R) \rightarrow H_{\mathfrak{m}}^{d}(A)$ being injective. Similarly, we have by assumption that the map $R / x R \rightarrow A / Q$ splits. So the map $\operatorname{Hom}_{R / x R}(A / Q, R / x R) \rightarrow \operatorname{Hom}_{R / x R}(R / x R, R / x R)$ is onto and, again by local duality, the map $H_{\mathfrak{m}}^{d-1}(R / x R) \rightarrow H_{\mathfrak{m}}^{d-1}(A / Q)$ is injective. To show that $x R \rightarrow Q$ splits we must prove that $H_{\mathfrak{m}}^{d}(x R) \rightarrow H_{\mathfrak{m}}^{d}(Q)$ is injective.

We have the following commutative diagram with exact rows.

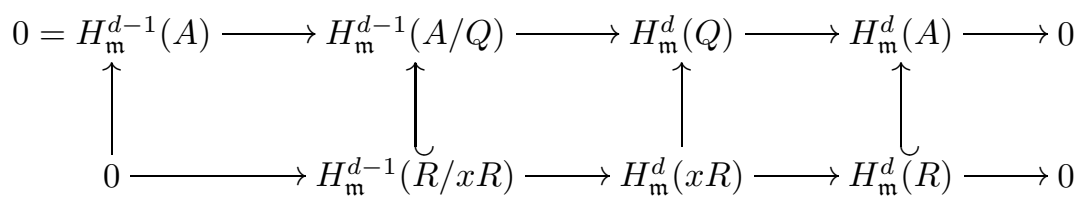

As in the previous proof, the maps $H_{\mathfrak{m}}^{d-1}(R / x R) \rightarrow H_{\mathfrak{m}}^{d-1}(A / Q)$ and $H_{\mathfrak{m}}^{d-1}(R) \rightarrow$ $H_{\mathfrak{m}}^{d}(A)$ are injective. Since $H_{\mathfrak{m}}^{d-1}(A)=0$, it follows that $H_{\mathfrak{m}}^{d}(x R) \rightarrow H_{\mathfrak{m}}^{d}(Q)$ is injective as well. Therefore $x R \hookrightarrow Q$ splits. 
Finally we recall two results of Dutta that illuminate special cases of the previous theorem. The first appears as Lemma 2.1 in [Dut97. The second is a rewording of Theorem 2.6 in Dut97.

Theorem 2.7 (Dutta). Let $A$ be a complete local almost complete intersection domain. Then $H_{\mathfrak{m}}^{d-1}(A)=0$.

Theorem 2.8 (Dutta). Let $A$ be a complete local normal domain such that its canonical module $\omega_{A}$ is $S_{3}$. Then $H_{\mathfrak{m}}^{d-1}(A)=0$.

Corollary 2.9. Let $A$ be a complete local domain of dimension $d$. Suppose the $D S C$ holds for rings of dimension $d-1$. Then the SDSC holds in the following cases:

(1) A is Cohen-Macaulay.

(2) $A$ is an almost complete intersection domain.

(3) $A$ is normal and $\omega_{A}$ is $S_{3}$, where $\omega_{A}$ denotes the canonical module of $A$.

Proof. In all three cases, $H_{\mathfrak{m}}^{d-1}(A)=0$. The result follows from Theorem 2.6 .

\section{ACKNOWLEDGEMENTS}

The author was supported in part by a University of Illinois Research Board grant and by NSA grant number H98230-06-1-0026, both awarded to Prof. Sankar Dutta. The author would like to thank the anonymous referee for many helpful suggestions and Sankar Dutta for his direction and support in numerous discussions.

\section{REFERENCES}

[Dut97] S. P. Dutta, Dualizing complex and the canonical element conjecture. II, Journal of the London Mathematical Society, Second Series, 56 (1997), no. 1, 49-63. MR1462825 (98g:13010)

[Dut98] A note on the monomial conjecture, Trans. Amer. Math. Soc. 350 (1998), no. 7, 2871-2878. MR 1466948 (98i:13047)

[Dut01] _ Splitting of local cohomology of syzygies of the residue field, Journal of Algebra 244 (2001), 168-185. MR1856533 (2002j:13023)

[EG81] E. Graham Evans and Phillip Griffith, The syzygy problem, Ann. of Math. (2) 114 (1981), no. 2, 323-333. MR632842 (83i:13006)

[Got83] Shiro Goto, On the associated graded rings of parameter ideals in Buchsbaum rings, J. Algebra 85 (1983), no. 2, 490-534. MR725097 (85d:13032)

[Hei02] Raymond C. Heitmann, The direct summand conjecture in dimension three, Ann. of Math. (2) 156 (2002), no. 2, 695-712. MR1933722 (2003m:13008)

[HH90] Melvin Hochster and Craig Huneke, Tight closure, invariant theory, and the BriançonSkoda theorem, Journal of the American Mathematical Society 3 (1990), no. 1, 31-116. MR.1017784 (91g:13010)

[HH93] , Phantom homology, Memoirs of the AMS, vol. 103, no. 490, American Mathematical Society, 1993. MR1144758 (93j:13020)

[HH95] _ Applications of the existence of big Cohen-Macaulay algebras, Advances in Mathematics 113 (1995), 45-117. MR.1332808 (96d:13014)

[Hoc73] Melvin Hochster, Contracted ideals from integral extensions of regular rings, Nagoya Mathematical Journal 51 (1973), 25-43. MR0349656 (50:2149)

[Hoc83] Canonical elements in local cohomology modules and the direct summand conjecture, J. Algebra 84 (1983), no. 2, 503-553. MR723406 (85j:13021)

[Hoc02] _ Big Cohen-Macaulay algebras in dimension three via Heitmann's theorem, Journal of Algebra 254 (2002), 395-408. MR1933876 (2004c:13011)

[Hoc07] - Homological conjectures, old and new, Illinois Journal of Math. 51 (2007), no. 1, 151-169. MR2346192(2008j:13034) 
[Koh86] Jee Koh, Degree $p$ extensions of an unramified regular local ring of mixed characteristic p, J. Algebra 99 (1986), no. 2, 310-323. MR837546 (87j:13027)

[Ran00] Nandini Ranganathan, Splitting in module-finite extension rings and the vanishing conjecture for maps of tor, Ph.D. thesis, University of Michigan, 2000.

[Rob76] Paul Roberts, Two applications of dualizing complexes over local rings, Ann. Sci. École Norm. Sup. (4) 9 (1976), no. 1, 103-106. MR0399075 (53:2926)

[SS93] Jan R. Strooker and Jürgen Stückrad, Monomial conjecture and complete intersections, Manuscripta Math. 79 (1993), no. 2, 153-159. MR1216771 (94c:13026)

[ZS93] Oscar Zariski and Pierre Samuel, Commutative algebra, vol. II, Graduate Texts in Mathematics, vol. 29, Springer-Verlag, 1993. MR0389876 (52:10706)

Department of Mathematics, University of Illinois at Urbana-Champaign, 273 AltGeld Hall, MC-382, 1209 W. Green Street, Urbana, Illinois 61801-2975

E-mail address: jmccullo@math.uiuc.edu 